\title{
THE BENEFITS OF ACTIVE REWARMING AFTER CARDIAC OPERATIONS: A RANDOMIZED PROSPECTIVE TRIAL
}

\author{
V. Pathi, FRCS ${ }^{\mathrm{a}}$ \\ G. A. Berg, $\mathrm{MD}^{\mathrm{a}}$ \\ J. Morrison ${ }^{\mathrm{a}}$ \\ G. Cramp, $\mathrm{MBChB}^{\mathrm{a}}$ \\ D. McLaren, FFARCS ${ }^{b}$ \\ A. Faichney, FRCS ${ }^{\mathrm{a}}$
}

\begin{abstract}
We conducted a prospective, randomized trial of three methods of rewarming patients after hypothermic cardiopulmonary bypass. Patients underwent either coronary artery bypass grafting or first-time valve replacement and were cooled to $32^{\circ} \mathrm{C}$ during bypass. No significant differences existed among the groups as regards operative or preoperative parameters including hemodynamics and blood use. The patients actively warmed with a convective (Bair Hugger system, 3 hours) or a conductive blanket (electric overblanket, 4 hours) reached normothermia more quickly than those warmed with the space blanket ( 7 hours). This was reffected in significantly earlier extubation in the former two groups: Bair Hugger system $10.8 \pm 0.6$ hours, electric blanket $11.3 \pm 1.0$ hours, and space blanket $14.8 \pm 0.8$ hours. Patients warmed with the space blanket required a higher dosage of morphine over the first 12 hours than those warmed with the electric blariket (10.4 vs $6.5 \mathrm{mg} ; p=0.004$ ), which may account for some of the differences between these two groups. No differences could be demonstrated between the two active blankets. On economic grounds we therefore recommend the reusable electric blanket for routine use. (J THORAC Cardiovasc Surg 1996;111:637-41)
\end{abstract}

Since the introduction of hypothermia into routine cardiopulmonary bypass (CPB), margins of safety for cardiac operations have greatly improved. ${ }^{1}$ Theoretically, the attenuation of the inflammatory response, ${ }^{2}$ reduced noncoronary collateral flow, and the longer "safe" circulatory arrest periods in case of catastrophies have made hypothermia an indispensible addition to the surgeons' armamentarium.

Despite being warmed systemically at the end of $\mathrm{CPB}$, patients invariably arrive in the intensive care unit with some degree of hypothermia. The time taken to reach normothermia passively is then dependent on the cardiac output, vascular resistance, body mass, surface area, and ambient temperature. External heat is another means of speeding up this process, but little work has been published to date

From the Departments of Cardiac Surgery and Anaesthesia, ${ }^{\mathrm{b}}$ Western Infirmary Glasgow, Glasgow, Scotland.

Received for publication Dec. 23, 1994; accepted for publication May 31, 1995.

Address for reprints: V. Pathi, MD, Department of Cardiac Surgery, Western Infirmary Glasgow, Dumbarton Rd., G11 6NT Glasgow, Scotland.

Copyright (C) 1996 by Mosby-Year Book, Inc.

$0022-5223 / 96 \$ 5.00+0 \quad \mathbf{1 2 / 1 / 6 6 6 0 2}$ on this aspect of postoperative care. Early attainment of normothermia not only would improve hemodynamics and gas exchange but also should improve hemostasis, which would lead to more rapid weaning from the ventilator.

We conducted a randomized prospective trial comparing three commonly used methods of rewarming patients in the intensive care unit. The aluminum foil blanket is the most commonly used method and theoretically acts by reflecting heat generated by the body, thus reducing loss to the environment. The electric filament blanket works by direct transfer of heat to the patient. The Bair Hugger convective heating system (Augustine Medical, Inc., Eden Prairie, Minn.) creates a microenvironment around the patient by pumping air of varying temperatures into a specially constructed blanket. Settings can be changed to meet requirements, ranging from $32^{\circ} \mathrm{C}$ to $42^{\circ} \mathrm{C}$.

\footnotetext{
Methods

Ninety patients undergoing hypothermic CPB for coronary artery grafting $(n=72)$ or valve replacement $(n=$ 18) were randomized into one of three groups. Group I had passive rewarming with an aluminum space blanket, group II active rewarming with a reusable electric overblanket, and group III active rewarming with the Bair Hugger convective warming system. The latter was set on
} 
Table I. Comparative patient data, with significance of $t$ test between groups

\begin{tabular}{lcccc}
\hline & Group I & Group II & Group III & $t$ Test \\
\hline Age (yr) & $61.6 \pm 1.6$ & $62.5 \pm 1.6$ & $61.8 \pm 1.3$ & NS \\
$\quad$ Range & $42-77$ & $46-79$ & $48-75$ & NS \\
Male:female & $18: 08$ & $20: 09$ & $19: 10$ & NS \\
Body surface area & $1.79 \pm 0.03$ & $1.8 \pm 0.03$ & $1.9 \pm 0.09$ & NS \\
Bypass time & $65 \pm 4$ & $69 \pm 3.3$ & $73 \pm 4.2$ & NS \\
Ischemic time & $40 \pm 2.7$ & $45 \pm 2.6$ & $46 \pm 3.1$ & NS \\
Blood loss (ml) & $637 \pm 55$ & $627 \pm 47$ & $605 \pm 60$ & NS \\
Blood usage (ml) & $135 \pm 37$ & $137 \pm 38$ & $163 \pm 42$ & NS \\
Colloid usage (ml) & $617 \pm 116$ & $667 \pm 161$ & $516 \pm 98$ & $10.7 \pm 0.6$ \\
Time to extubation (hr) & $14.8 \pm 0.8$ & $11.3 \pm 1.0$ & & $p=0.0002, *$ \\
& & & $p=0.009 \dagger$ \\
\hline
\end{tabular}

All parameters are given as mean \pm standard error of the mean. NS, Not significant. $(p>0.05)$,

*Group I versus group III.

†Group I versus group II.

high until the core temperature reached $37^{\circ} \mathrm{C}$ and was then reduced to low.

$\mathrm{CPB}$ was conducted at $32^{\circ} \mathrm{C}$ with a Stockert-Shiley roller pump (Shiley, Inc., Irvine, Calif.) and a hollow fiber membrane oxygenator (Bard HF 5700, Bard Cardiopulmonary Division, Tewksbury, Mass.). Crystalloid prime was used in all cases and full flows were maintained at a rate of $2.4 \mathrm{~L} / \mathrm{m}^{2}$. Myocardial protection was with multidose cold crystalloid cardioplegic solution (St. Thomas' Hospital solution). Anesthesia was induced with fentanyl 10 to $20 \mu \mathrm{g} / \mathrm{kg}$ and etomidate 0.2 to $0.4 \mathrm{mg} / \mathrm{kg}$ and maintained with enflurane and propofol during CPB. Pancuronium was used for muscle relaxation.

Patients were warmed to $37^{\circ} \mathrm{C}$ during CPB and transferred to the intensive care unit before the appropriate blanket was used. All patients were given a glycerin trinitrate infusion (10 to $50 \mu \mathrm{g} / \mathrm{kg}$ per minute.) If hypertension was a problem after the operation, the infusion rate of glycerin trinitrate was increased and analgesic and sedative drugs were administered if appropriate. Resistant hypertension was managed by an infusion of sodium nitroprusside, titrated to maintain a systolic blood pressure of 110 to $130 \mathrm{~mm} \mathrm{Hg}$. Hypotension unresponsive to optimization of filling pressures was initially managed by increasing doses of dopamine to $15 \mu \mathrm{g} / \mathrm{kg}$ per minute. If this was inadequate, epinephrine infusion was begun. Sequential determinations of blood pressure and urine output were used to titrate the doses of inotropic drugs. Mean blood pressures below $50 \mathrm{~mm} \mathrm{Hg}$ were treated by increasing the inotropic agents.

Morphine was primarily used for analgesia in incremental doses of 2 to $4 \mathrm{mg}$, with 1 to $2 \mathrm{mg}$ of midazolam for sedation. If clinically apparent shivering was noted, $2 \mathrm{mg}$ doses of vecuronium bromide were given. Propofol infusion was used if these measures were believed to be inadequate and prolonged ventilatory assistance was believed to be necessary.

During the first 18 hours after the operation, routine hemodynamic parameters were monitored, including heart rate, arterial blood pressure, central venous pressure, urinary output, blood loss, and blood and colloid replacement. Core temperature was measured with a nasopharyngeal probe and peripheral temperature was measured at the big toe. These temperatures are routinely used to monitor cooling and rewarming during CPB in our unit. Swan-Ganz thermodilution cardiac output catheters (Baxter Healthcare Corp., Edwards Div., Santa Ana, Calif.) were inserted only if difficulty was experienced in weaning patients from $\mathrm{CPB}$ and particularly to monitor the introduction of mechanical or inotropic support. Patients were extubated when they were in hemodynamically stable condition, not bleeding, fully rewarmed, breathing spontaneously, and obeying commands.

Results are presented as mean \pm standard error of the mean. Statistical analysis was performed with the unpaired Students' $t$ test on software of Microsoft Excel version 5.0 (Microsoft Corporation, Redmond, Wash.), and significance was accepted at a $p$ value less than 0.05 .

\section{Results}

Eighty-four patients completed the study protocol. Because of the nature of the treatments, blinding of the nursing staff to the protocol was impossible and may have introduced an element of bias into the study. Four patients were withdrawn because of poor cardiac output necessitating inotropic or mechanical support and long term mechanical ventilation for more than 48 hours. Two others were excluded because of surgical bleeding necessitating rethoracotomy. Group I comprised 26 patients, group II 29 , and group III 29 . They were well matched for age and sex. There were no significant differences in any preoperative or operative patient characteristics (Table I). No statistically significant intergroup differences were found for the mean hourly values of heart rate, blood pressure, central venous pressure, urinary output, inspired percentage of oxygen, or arterial partial pressures of oxygen or carbon dioxide (smallest $p$ value on $t$ test was 
Table II. Analgesic, sedative, muscle relaxant, and vasoactive drug use during the first 12 hours

\begin{tabular}{lcccc}
\hline & Group I & Group II & Group III & t Test \\
\hline Morphine (mg/12 hr) & $10.4 \pm 1.1$ & $6.5 \pm 0.8$ & $8 \pm 0.8$ & $p=0.004^{*}$ \\
Morphine (mg/6 hr) & $5.4 \pm 1.2$ & $4.4 \pm 0.7$ & $5.6 \pm 0.8$ & $p>0.1$ \\
Midazolam (mg/12 hr) & $7.2 \pm 0.8$ & $5.6 \pm 0.7$ & $5.7 \pm 0.7$ & $p>0.1$ \\
Propofol (No. of patients) & 2 & 1 & 3 & \\
Vecuronium (No. of patients) & 3 & 1 & 2 & 1 \\
Epinephrine (No. of patients) & 0 & 1 & 2 & \\
Nitroprusside (No. of patients) & 4 & 3 & \\
\hline
\end{tabular}

*Group I versus group II.

0.12). Similarly, the mean hourly infusion rates of nitrate or dopamine failed to differ significantly, although the trend was toward a higher mean dopamine infusion rate at 2 to 3 hours in group III than in group I ( 2 hours: $4.2 \mu \mathrm{g} / \mathrm{kg}$ per minute in group III vs $3.9 \mu \mathrm{g} / \mathrm{kg}$ per minute in group I, $p=$ $0.08,3$ hours: $3.9 \mu \mathrm{g} / \mathrm{kg}$ per minute in group III vs $3.6 \mu \mathrm{g} / \mathrm{kg}$ per minute in group $\mathrm{I}, p=0.10$ ). Epinephrine infusion was used in two patients only and nitroprusside in nine (Table II). These patients were evenly distributed among the groups. Sedative and muscle relaxant use was equal between the groups, but there was a significantly higher use of morphine over the first 12 hours in group I than in group II $(10.4 \pm 1.1 \mathrm{mg}$ vs $6.5 \pm 0.8 \mathrm{mg} ; p=0.004)$, with a similar trend when compared with group III $(8.0 \pm$ $0.8 \mathrm{mg} ; p=0.08$ ).

In the postoperative phase, although there was a trend toward higher blood use (amounting to $30 \mathrm{ml}$ per patient) and lower colloid requirements in patients warmed with the Bair Hugger system $(150 \mathrm{ml}$ per patient), these differences did not reach statistical significance (see Table I). The major differences seen were in the rate of rewarming in the three groups, with the active methods being far superior. The Bair Hugger system was significantly better at 2 hours and the electric blanket at 3 hours (Fig. 1). The space blanket took an extra 4 hours to fully warm the patients. When the peripheral temperatures were examined, the Bair Hugger system was the only method not to display the early decrease seen with the other two blankets (Fig. 2). This difference was statistically significant from the outset and lasted until the fifth hour. The time to extubation was significantly shorter in the two actively warmed groups (Table I). There was no difference between the two active warming methods, but the patients were extubated 3.5 hours (electric blanket) and 4.1 hours (Bair Hugger system) earlier than the space blanket group.

\section{Discussion}

It is widely believed that early rewarming after hypothermic CPB is desirable to expedite recovery. The recent trend toward warm heart surgery pioneered in Toronto ${ }^{3,4}$ does show major benefits from early rewarming in terms of shorter duration of ventilatory support. This method, however, has not gained widespread popularity. In a recent survey of CPB procedures in Australia, all units used some degree of cooling during cardiac operations. ${ }^{5}$ This probably also reflects the majority view in Europe and the United States.

In a recent study of patients after heart operations, Mort, Rintel, and Altman ${ }^{6}$ found reduced shivering and oxygen consumption if convective methods of warming were used in the postoperative period. Their requirements for sedative and muscle relaxant drugs were also reduced in this study. The prevalence of shivering was minimal in our study and warranted treatment in only six patients, with none of the methods being immune. This low incidence of shivering may have been due to the patients being cooled to only $32^{\circ} \mathrm{C}$ during CPB. Their core temperatures were on average just under $35^{\circ} \mathrm{C}$ on return to the intensive treatment unit. The hangover effect of the muscle relaxants also may have contributed to this low level of shivering; in previous published studies, extended rewarming during $\mathrm{CPB}$ to core temperatures of $38.5^{\circ} \mathrm{C}$ has been shown to reduce though not abolish the shivering response. ${ }^{7}$

The more rapid rewarming by active means is in general agreement with other published reports. ${ }^{8,9}$ These have shown that both core and peripheral temperatures reach extubation criteria more rapidly, although whether patients were in fact extubated earlier is not clear. The use of a radiant heat source from the ceiling was also beneficial in the studies published by Joachimsson, Nystrom, and Tyden. ${ }^{10,11}$ In these patients cooled to $25^{\circ} \mathrm{C}$, reduced 


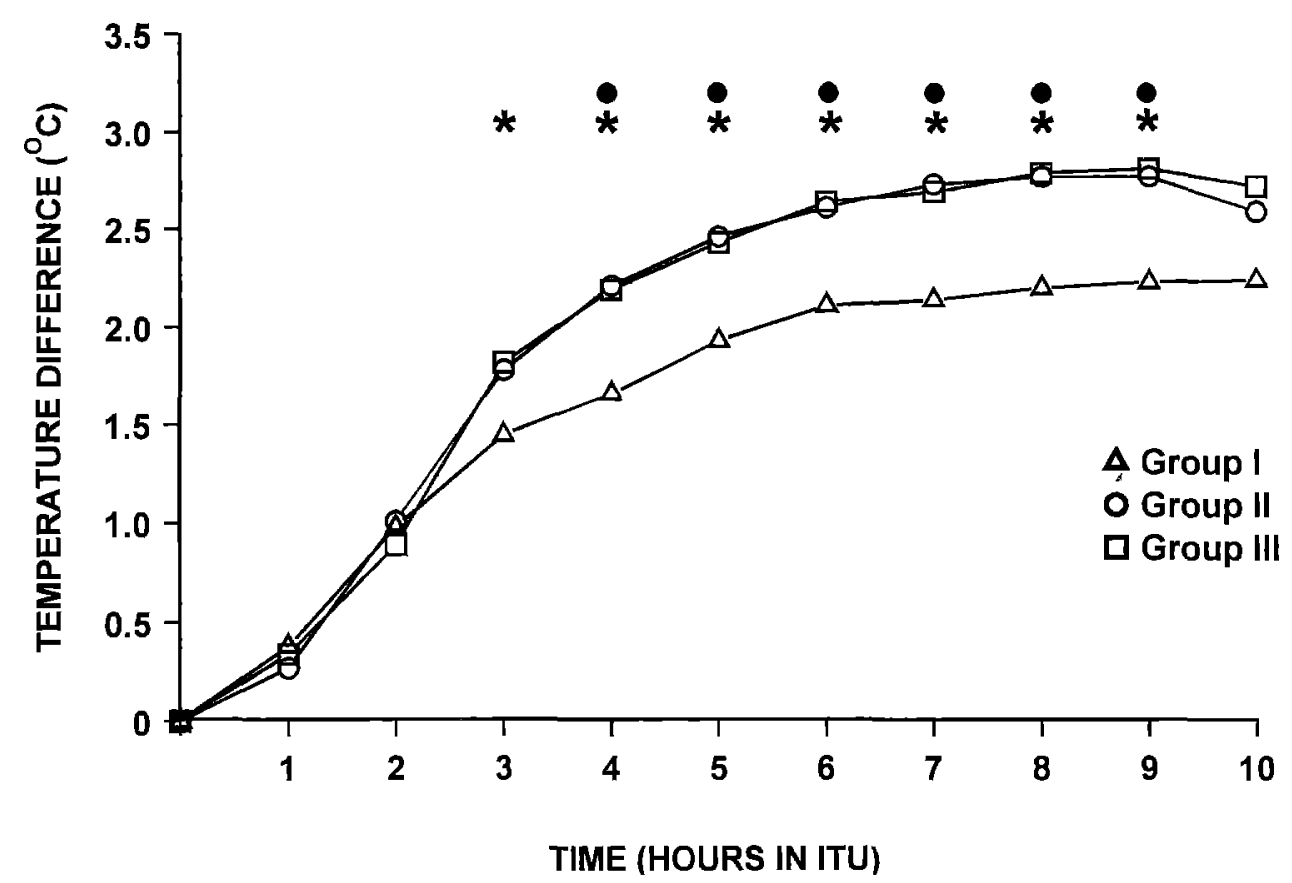

Fig. 1. Mean hourly changes in nasopharyngeal temperature over the first 10 hours. Unpaired $t$ test: * group I versus III, $p<0.05$; group I versus II, $p<0.05$. ITU, Intensive treatment unit.

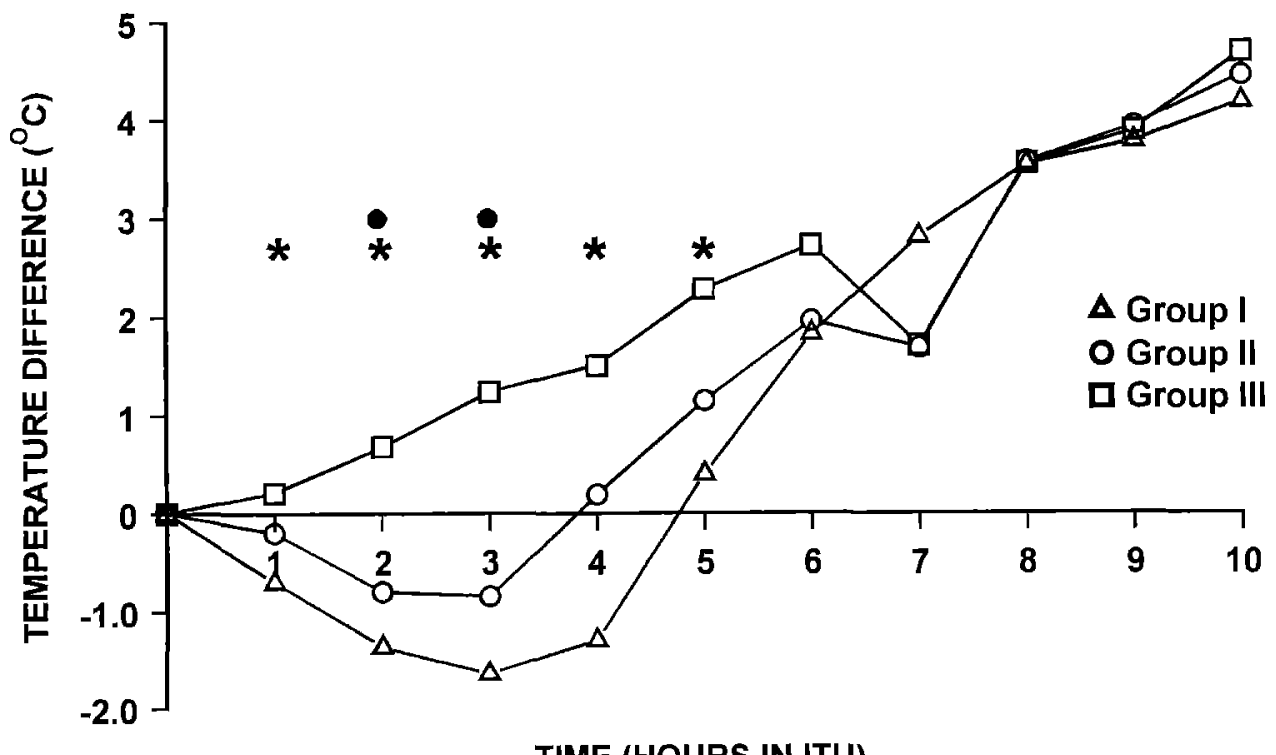

TIME (HOURS IN ITU)

Fig. 2. Mean hourly changes in big toe temperatures. Unpaired $t$ test: "group I versus III, $p<0.05$; group I versus II, $p<0.05$. ITU, Intensive treatment unit.

oxygen consumption and carbon dioxide production, lower shivering scores, and more stable hemodynamics were found. Our patients who were warmed actively reached extubation temperatures at between 3 and 4 hours, whereas those warmed pas- sively took a further 4 hours. Although hemodynamic parameters measured were not statistically different, use of dopamine tended to be more common in group III than in group I. This difference did not reach statistical significance; however, the nurs- 
ing staff would be expected to administer dopamine to control the fall in blood pressure caused by vasodilation induced by the convective warming system. Similarly, the increased trend of blood use but lower colloid replacement could be explained by the reduction in capillary permeability in the warmer group, leading to retention of more fluid intravascularly and thus hemodilution. This would be registered as a low hemoglobin value, which would prompt blood rather than colloid transfusion.

Inasmuch as there were no significant differences in hemodynamics, blood loss, or gas exchange, it can only be postulated that the metabolism of anesthetic agents is improved at these temperatures, allowing the patient to cooperate fully in his or her own ventilation. The doses of morphine administered over the first 6 hours were not significantly different among the groups, although the cumulative doses over 12 hours were statistically higher in the patients warmed with space blanket (see Table II). On questioning patients while they were still intubated and after extubation, we learned that the majority waking up at lower temperatures expressed a feeling of discomfort and lethargy and a greater awareness of pain, which disappeared after a dose of opiate. When they awoke a second time at normothermia, they felt much more able to tolerate spontaneous ventilation and were then extubated. This would obviously have a bearing on extubation times because patients waking up during this period may not have been fully cooperative and fit for extubation until full rewarming had been achieved. This was the predominant pattern of events seen leading to delayed extubation.

Although the differences were statistically significant, the extubation times in our groups varied only by 3 and 4 hours, which does not translate into significant differences in cost efficiency. The potential benefits to patients not restricted to being cooled to only $32^{\circ} \mathrm{C}$ may be more obvious. Published reports of early extubation of patients have stressed the importance of savings accrued from shorter intensive care stay together with the productivity loss caused by the "intensive care unit gridlock" scenario, with cancellation of cases because of bed shortage. ${ }^{12,13}$

The comparative costs of the three blankets show that the space blanket is the cheapest ( $£ 1.05, \$ 1.72$ ) and the Bair Hugger system the most expensive (£12.40, \$20.30). The initial outlay for the convective heating unit is $£ 1275$ (\$2091), whereas the electric blankets retail at $£ 42$ ( $\$ 68.90$ ) each but can be safely used for more than a year. The theoretic disadvantage of infection transfer has not been borne out in our experience, and no burns to the patient have been noted. The practice of enclosing the electric blanket in normal cotton sheets may be important in this context and does not impede heat transfer. It would therefore seem that the reusable electric blanket is the most cost-effective form of rewarming in this group of patients.

\section{REFERENCES}

1. Bigelow WG, Lindsay WK, Greenwood WF. Hypothermia: its possible role in cardiac surgery. Ann Surg 1950;132:84966.

2. Moore FD, Warner KG, Assousa S, Valeri CR, Khuri SF. The effects of complement activation during cardiopulmonary bypass: attenuation by hypothermia, heparin and hemodilution. Ann Surg 1988;208:95-103.

3. Christakis GT, Koch JP, Deemar KA, et al. A randomized study of the systemic effects of warm heart surgery. Ann Thorac Surg 1992;54:449-59.

4. Page RD, Sharpe DA, Bellamy CM, Rashid A, Fabri BM. Normothermic arrest with continuous hyperkalaemic blood: initial experience. Eur J Cardiothorac Surg 1992;6:461-8.

5. Wajon PR, Walsh RG, Symons NLP. A survey of cardiopulmonary bypass perfusion practices in Australia in 1992. Anaesth Intensive Care 1993;21:814-21.

6. Mort TC, Rintel TD, Altman F. Shivering in the cardiac patient: evaluation of the Bair-Hugger warming system. Anesthesiology 1990;73(3A):A239.

7. Joachimsson PO, Nystrom SO, Tyden H. Postoperative ventilatory and circulatory effects of extended rewarming during cardiopulmonary bypass. Can J Ariaesth 1989;36:9-19.

8. Cross MH, Davies JC, Shah MV. Post operative warming in the cardiac patient: evaluation of the Bair-Hugger convective warming system. Abstract presented at the EACTA meeting, Finland, 1994.

9. Moors AH, Pickett JA, Bethune DW, Duthie DJR. Convective warming following hypothermic cardiopulmonary bypass. Abstract presented at the European Association of CardioThoracic Anesthetists meeting, Finland, 1994.

10. Joachimsson PO, Nystrom SO, Tyden H. Heating efficiency of external heat supply during and after open heart surgery with hypothermia. Acta Anaesthesiol Scand 1987;31:73-80.

11. Joachimsson PO, Nystrom SO, Tyden H. Postoperative ventilatory and circulatory effects of heating after aortocoronary bypass surgery: postoperative external heat supply. Acta Anaesthesiol Scand 1987;31:532-42.

12. Quasha AL, Loeber N, Feeley TW, et al. Postoperative respiratory care: a controlled trial of early and late extubation following coronary artery bypass grafting. Anesthesiology 1980;52:135-41.

13. Higgins TL. Early endotracheal extubation is preferable to late extubation in patients following coronary artery surgery. $\mathbf{J}$ Cardiothorac Vasc Anesth 1992;6:488-93. 